\title{
Demandas técnico-táticas e fisiológicas de combates da luta olímpica
}

\author{
Bianka MIARKA* \\ Escola Superior de Educação Física, Universidade Federal de Pelotas (Brasil)
}

Recepción: 15/12/2015; Aceptación: 05/04/2016; Publicación: 21/06/2016.

\begin{abstract}
Resumo
Análises das demandas técnico-táticas e fisiológicas dos combates da luta olímpica são importantes porque mostram informações essenciais para o desenvolvimento de treinamentos contextualizados e preparação física específica para lutadores. Por isso, o objetivo dessa revisão é mostrar características do combate de luta olímpica nos estilos livre, feminino e greco-romana. A análise de tempo-movimento apresentada nesse artigo é componente principal para inferências sobre a intensidade em relação à taxa de esforço: pausa pelas ações do combate. Após a modificação das regras durante o ano de 2013, os combates mostraram ações rápidas e com maior variação, especialmente, em categorias mais leves. Durante os combates de greco-romano, grande parte dos estudos apontam os takedowns como as técnicas mais eficazes em competições mundiais entre 2009 e 2011. Para o mesmo período, na luta olímpica feminina e estilo livre, as técnicas de pé/perna são as mais eficazes, seguida dos ataques de takedown. Enquanto, as últimas análises, após a modificação das regras, mostram que as ações ofensivas determinantes são aplicadas em pé e em par-terre, especialmente técnicas de gutwrenches e movimentos derivados do suplex. Os conhecimentos sobre as ações determinantes e predominantes dos combates da luta olímpica podem ser aplicados em futuras pesquisas, assim como utilizados em aplicação prática em treinamentos, preparação física e avaliações análogos às ações do combate, assim como permitem intervenções para evitar lesões que possam ser conseqüentes das ações técnico-táticas da luta olímpica.
\end{abstract}

ORIGINAL PAPER

Keywords: Artes marciais; wrestling; técnica; tática; comportamento motor; análise de tempo-movimento; esportes de combate.

\section{Demandas técnico-tácticas y fisiológicas de combates de lucha olímpica}

\section{Resumen}

El análisis de las demandas técnico-tácticas y fisiológicas de los combates de lucha olímpica es importante ya que aporta información esencial para el desarrollo de entrenamientos contextualizados y para la preparación física específica de los luchadores. Por ello, el objetivo de esta revisión es mostrar las características del combate de lucha olímpica en los estilos libre, femenina y grecorromana. El análisis de tiempomovimiento que se presenta en este trabajo es el componente principal para poder realizar inferencias sobre la intensidad en relación al esfuerzo: relación entre las pausas y las acciones del combate. Tras las modificaciones de las reglas en el 2013, los combates mostraron acciones rápidas y con una mayor variabilidad, especialmente en categorías más ligeras. Durante los combates de lucha grecorromana gran parte de los estudios apuntan a los takedowns como las técnicas más eficaces en competiciones mundiales entre los años 2009 y 2011. Para el mismo período, en lucha olímpica femenina y el estilo libre, las técnicas de pié/pierna son las más eficaces, seguidas de los ataques de takedown. Los últimos análisis, tras las modificaciones de las reglas, muestran que las acciones ofensivas determinantes son aplicadas en pie y en par-terre, especialmente técnicas de gutwrenches y movimentos derivados de suplex. Los conocimientos sobre las acciones determinantes y predominantes en los combates de lucha olímpica pueden ser utilizados en futuras investigaciones, así como aplicarse de manera práctica en entrenamientos, preparación física y evaluaciones análogas de las acciones del

\section{Technical-tactical and physiological demands of wrestling combats}

\section{Abstract}

Technical-tactical and physiological demand analyses of wrestling combats are important because they reveal essential information for the development of contextual training and specific physical preparation of wrestlers. Therefore, the aim of this review is to describe the characteristics of wrestling combats in freestyle, female and Grecoroman styles. The time-motion analysis presented in this article is the main component to carry out inferences on intensity and effort: pause ratio of combat actions. After rules modification in 2013, wrestling combats showed quicker and more diversified actions, especially in the lighter categories. For Greco-roman wrestling, most studies showed takedowns as the most effective techniques in World competitions for the period 2009-2011. For the same period, foot/leg techniques were the most effective in female and freestyle wrestling, followed by takedown attacks. Recent analyses, developed after 2013 rules modification, showed that the decisive offensive actions were applied on foot and in par terre situations - especially gutwrenches techniques and derivatives of suplex movements. The knowledge on the determinant and predominant actions of wrestling combats can be applied in future research, as well as can be used practically for training, physical preparation and assessments of

*E-mail: miarkasport@hotmail.com 
combate. También posibilitan realizar intervenciones para evitar lesiones que puedan ser consecuencia de las acciones técnico-tácticas de la lucha olímpica.

Palabras clave: Artes marciales; lucha; técnica; táctica; comportamiento motor; análisis de tiempo-movimiento; deportes de combate. similar combat actions. It also allows interventions to prevent injuries resulting from technical and tactical wrestling actions.

Palavras-chave: Martial arts; wrestling; technique; tactic; motor control; time-motion analysis; combat sports.

\section{Introdução}

É consenso entre os especialistas que o sucesso em combinações técnico-táticas em combates está vinculado à prática contextualizada nos treinamentos e ao conhecimento dos resultados das ações desempenhadas nas competições; treinos mais eficazes devem simular situações competitivas (Barna, 2013; Cipriano, 1993; López-González, 2014; Miarka, Julio, Del Vecchio, Calmet, \& Franchini, 2010). A importância desses fatores permite mais ênfase sobre as análises notacionais em competições de luta olímpica, sobretudo em razão da estrutura temporal e técnico-tática (Barna, 2013; López-González \& Miarka, 2013). Além de proporcionar informações que contribuem para a interpretação do esforço específico requerido, esse tipo de observação permite inferir o plano estratégico escolhido por atletas de elite, de acordo com as demandas do combate (Cipriano, 1993; López-González, 2011; Tünnemann, 2012). Porém, em virtude de fatores como categoria de peso, gênero e das mudanças que ocorrem nas regras ao longo do tempo, conseqüentemente, as ações durante o confronto também modificam. Por sua vez, isso interfere no conhecimento de resultados e em observações das possíveis correlações existentes na execução de combinações e padrões de ação de torneios da luta olímpica para aplicação em treinamentos contextualizados (Barna, 2013; Miarka \& Conceição, 2012).

Há algumas décadas, porém, desde estudos pioneiros do meio do século $\mathrm{XX}$, as análises notacionais de ações técnicas em combates com características de preensão manual e domínio sobre o oponente têm sido realizadas (Tünnemann, 2011a). Na década de 1970, a técnica era caracterizada como elemento fundamental e básico na configuração e no desenvolvimento das ações no combate. No entanto, uma das evoluções mais importantes na história dos estudos notacionais na luta olímpica, bem como nos conseqüentes procedimentos de análise, foi considerar a tática, e não apenas a técnica, fator essencial no confronto e no desempenho dos atletas (Tünnemann, 2012). Apesar de as pesquisas atuais ainda realizarem análises isolando variáveis técnico-táticas - dada a escassez de metodologias que permitam verificar a interação entre as ações -, já se sabe que a luta olímpica não é apenas um conjunto de situações técnico-táticas fragmentadas. Os atletas combinam ações para originar diferentes arranjos, objetivando efeito favorável no resultado do combate (López-González, 2014).

Para as competições de luta olímpica no masculino os torneios olímpicos são no estilo greco-romano e no estilo livre, ambos os estilos são tarefas abertas que exigem combinações complexas entre o momento de domínio por preensão manual e a aplicação da técnica. Essa interação entre os atletas é uma variável essencial para o comportamento técnico-tático das modalidades esportivas com ações classificadas como tarefa aberta (Lafon, 2008). Segundo as regras, no estilo greco-romano os atletas podem realizar o controle do oponente com preensão manual apenas acima da linha da cintura e as ações de ataque e defesa só podem ser aplicadas com a utilização dos braços e do tronco. Para o estilo livre, as ações de ataque podem ser realizadas em qualquer parte do corpo do oponente, desde que não seja considerada perigosa. Tanto na grecoromana, quanto no estilo livre investigar as possíveis combinações é crucial para o estudo da tática relacionada à luta olímpica, pois a oposição direta entre os atletas faz que a ação de cada indivíduo se torne um estímulo para uma possível resposta do adversário (López-González \& Alonso, 2011). As competições para o estilo livre feminino foram incluídas apenas nos Jogos Olímpicos de Atenas em 2004, para atender um princípio estabelecido pelo Comitê Olímpico Internacional, que determina a igualdade de gênero quanto à participação no esporte (FILA, 2015). 0 feminino é disputado apenas no estilo livre e, para todos os estilos e gêneros, o objetivo formal do combate é realizar o assento, chamado também de encostamento, que é definido pela colocação das costas do adversário no tapete pelo tempo suficiente para o árbitro observar o total controle sobre o oponente e, no estilo livre, pode ocorrer o encostamento instantâneo, que acontece quando o 
lutador encosta ambos os ombros do adversário no tapete por pelo menos 1 segundo. Isso não é considerado um assento tradicional, porém o lutador que atacou recebe 4 pontos, se demonstrar controle parcial sobre o oponente e não total, como no encostamento tradicional (FILA, 2015).

O tempo dos rounds, assim como das ações pode variar em função da idade, gênero e estilo, sendo que, caso a imobilização por um dos atletas não seja alcançada ao final do tempo regulamentar, a decisão do vencedor do combate é baseada em uma pontuação variável para as ações técnicas ofensivas e/ou defensivas e sanções disciplinares (FILA, 2015). As ações e projeções executadas pelos atletas durante os combates recebem pontuações que são classificadas por um, dois, três e cinco pontos. Ao alcançar a diferença de seis pontos com relação ao seu adversário, o atleta é considerado vencedor do round por superioridade técnica (FILA, 2015).

Apesar da importância, na análise, das adaptações técnico-táticas - que podem ser extremamente rápidas -, com o executante eficaz apresentando várias opções de resposta para um mesmo estímulo, são ainda escassos os estudos de revisão sobre variáveis técnico-táticas e o estabelecimento de padrões e variações na interação entre o executante e o oponente para obter pontos durante o confronto. Investigações mais recentes apontam a variação técnica como elemento fundamental para a imprevisibilidade em competições de alto rendimento (Miarka, et al., 2010). Logo, a eficácia das ações motoras depende da tomada de decisão no menor intervalo de tempo possível e de acordo com as imposições e exigências emergentes do combate.

A fim de caracterizar e justificar o presente estudo sobre a caracterização técnico-tática de combates de luta olímpica, a revisão de literatura analisa de forma meticulosa as investigações sobre o assunto e descreve os resultados de forma crítica, abordando problemas metodológicos e conceituais. Além disso, são apresentados conceitos, utilizados para a elaboração do modelo de estrutura temporal e técnico-tática validado por López-González e Miarka (2013), advindos de estudos prévios, com foco nas observações técnico-táticas da estrutura temporal do combate. Portanto, o objetivo é realizar uma caracterização do combate de luta olímpica nos estilos livre e greco-romana para serem utilizados como fonte de informação na planificação de treinamentos.

\section{Caracterização do combate}

Baseadas em regras, os confrontos de um torneio são realizadas em eliminatória direta, com repescagem para os perdedores dos lutadores classificados para final. Cada categoria é disputada em um único dia, sendo que a pesagem é realizada no dia anterior aos combates (FILA, 2015). Após as pesagens, os lutadores são seriados em função da idade, categoria de peso e gênero. Especificamente, segundo o regulamento oficial da Confederação Brasileira de Lutas Associadas (2013), a duração dos combates diferencia em função da idade. Atletas escolares e infantis realizam dois rounds de 1 ou 2 min cada. Cadetes, juniores e seniores no estilo livre combatem três rounds de 2 min com 30 s de descanso. Porém, se ao término do round do combate do estilo livre o placar for 0 versus 0 , o combate é prolongado por mais 30 segundos, reiniciando-se na posição declinch. No estilo greco-romano os atletas lutam três rounds de 1 min por dois tempos de $30 \mathrm{~s}$ para o clinch, o que totaliza 2 min de combate, com $30 \mathrm{~s}$ de intervalo entre esses períodos. Ao final de cada round o árbitro deve declarar o vencedor, se o lutador vencer dois rounds ele ganha o combate. Caso ocorra o empate do round, o vencedor é determinado pelos pontos técnicos e se ainda ocorrer a equidade, o último ponto técnico determinará quem vence o round (FILA, 2015).

Medalhistas internacionais realizam cerca de 5 combates por competição, com um tempo mínimo de 15 min para recuperação entre elas (Kraemer, et al., 2001; Barbas, et al., 2011). Conceitualmente, distinguir as características dos combates de luta olímpica é uma forma de obter informações relevantes para organizar a aplicação dos modelos de periodização na preparação física e técnico-tática. Para os três estilos de luta (greco-romano, livre e feminina) as regras e a forma de pontuar são as mesmas, exceto no estilo greco-romano, no qual não é permitido utilizar os membros inferiores para realizar ataques e/ou defesas (López-Gullón \& Martínez-Abellán, 2011). 0 objetivo principal da luta olímpica para vitória é realizar o assentamento do adversário com as costas no solo durante pelo menos 3 segundos, ou seja, realizar o touch. Além do encostamento, também é possível pontuar através de projeção (3 até 5 pontos), retirada da área de competição (1 ponto), domínio parcial do adversário pelas costas (1 ponto) e pelo domínio completo do oponente 
pelas costas no solo ( 2 pontos). No caso, quem obtiver 6 pontos de diferença ou realizar uma projeção de 5 pontos ganha o round. Se um atleta conquistar a vitória de dois rounds, automaticamente ganha o combate por superioridade técnica (FILA, 2015).

Tanto na luta livre, quanto na greco-romana, em relação às ações que pontuam, pesquisas com estrutura temporal e análise notacional buscaram descrever como os atletas atuam no período de combate para obter o touch (Tünnemann, 2011a,b,c). Em grande parte dos casos, os confrontos estão sendo decididos por pontos conquistados com outros meios ofensivos, como retirada da área de combate e domínio parcial das costas, em razão da dificuldade para realizar o assentamento do adversário no solo (Tünnemann, 2011a,b,c). Dados estatísticos revelaram que menos de 2\% dos combates foram conquistados pelo touch nos Jogos Olímpicos de Londres, 2012 (FILA, 2015). A dificuldade em pontuar aumenta à medida que a categoria de peso corporal é mais elevada, conforme mostra a Figura 1.

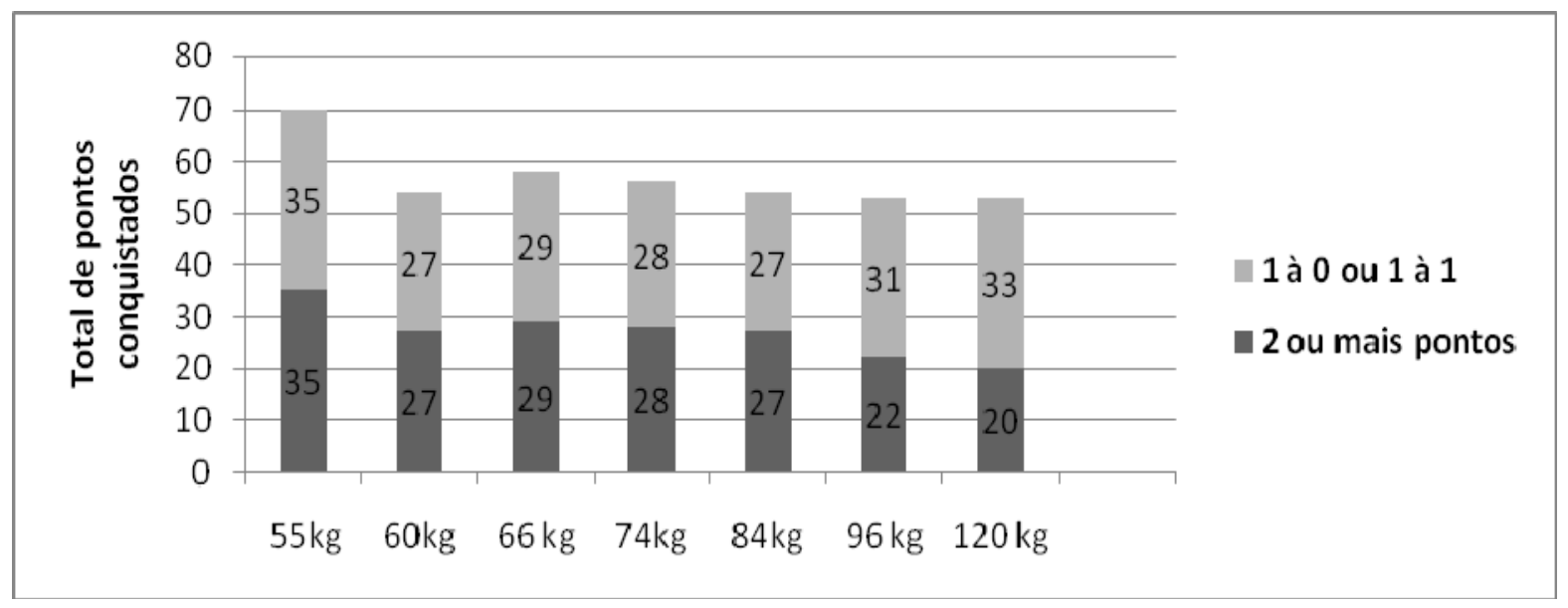

Figura 1. Gráfico de pontuações: 2 ou mais pontos por round versus placar de 1 a 0 ou 1 a 1 por round, em combates do estilo greco-romana nos Jogos Olímpicos de Londres, 2012, separados por categoria de peso

(dados adaptados de Tünnemann, 2013).

A Figura 1 revela que atletas da categoria $55 \mathrm{~kg}$ realizaram $50 \%$ da pontuação com contagem de 2 ou mais pontos, em contrapartida, atletas do pesado obtiveram apenas 38\% dos pontos nessa situação. Tal característica indica que categorias mais leves possuem maior probabilidade de conquista do round por superioridade técnica. Enquanto, provavelmente, atletas mais pesados obtiveram maior número de pontos por situações de saída de área e punição pela passividade ou trava do combate, o que sugere maior uso de preensão manual, na tentativa de controlar o oponente e maior freqüência de recomeço em posição par-terre (Tünnemann, 2012). Essa especificidade técnico-tática para conquista de vitória no combate indica a premissa de que esses elementos são componentes determinantes para conquista da vitória na luta olímpica.

Desse modo, as caracterizações do combate devem ser utilizadas para melhora do desempenho através do implemento de atividades com habilidades específicas da modalidade no treinamento dos atletas (Curby, 2009; Kirov \& Makavéev, 2010). Por exemplo, o Programa USOEC qualificou e combinou ações do estilo livre e greco-romano para avaliar e preparar lutadores norteamericanos ao longo da temporada na estruturação, controle e organização do treino de acordo com o calendário competitivo (Curby, 2005).

Pioneiramente, Matveev implantou a planificação do tipo linear polifacetada, cuja meta foi desenvolver aspectos físicos, psicológicos, técnicos e táticos, de acordo com a condição do atleta e do calendário de competição (Kirov \& Makavéev, 2010). Além desse modelo, outras planificações lineares e não lineares implementaram o ajuste de intensidade e volume de treino para elevar a performance frente à constante modificação e ao elevado número de competições do calendário competitivo (Kraemmer, et al., 2000; Rhea, Ball, Phillips, \& Burkett, 2002), como pode ser visto no exemplo da Tabela 1.

Em razão do alto número de competições, como mostra a Tabela 1, técnicos e preparadores físicos passaram a utilizar torneios menos importantes como meio de treinar e até mesmo como 
forma de avaliar os aspectos abordados durante o treinamento para os campeonatos principais (Gomes, 2002). Esse monitoramento durante a competição otimiza o processo de preparação e pode ser realizado através da quantificação de ações e avaliação das reações imediatas do organismo por meio das respostas fisiológicas (Barbas, et al., 2011; Horswill, 1992; Kraemer, et al., 2001; Platonov, 2004; Yoon, 2002). Por exemplo, diferentes pesquisas sobre o esforço em competições demonstraram que tanto as concentrações de cortisol, quanto a norepinefrina e a adrenalina aumentam significativamente após cada confronto $(p \leq 0,05)$ em cinco combates competitivos com perda de peso prévia do estilo livre e do greco-romano (Barbas, et al., 2011; Kraemer, et al., 2001).

Esses conhecimentos sobre as competições trazem importantes indicativos neuromusculares e metabólicos, associados ao desempenho (Horswill, 1992; Yoon, 2002). Especialmente, quando os dados ligam-se: i) com as diferentes manifestações de força muscular (força isométrica, resistência e potência) - determinantes para ações de tentativa de domínio do oponente, defensivas e ataques rápidos, e ii) ao metabolismo, prevalente e determinante, e com a demanda de fadiga - em resposta à intensidade e volume de ações em combate (Horswill, 1992; Yoon, 2002).

Tabela 1. Calendário da Confederação Brasileira de Lutas Associadas de 2012 (Confederação Brasileira de Lutas Associadas, 2013).

\begin{tabular}{|c|c|c|c|}
\hline Data & Local & Evento & Estilo \\
\hline JAN2-12 & CEFAN- RJ & Campo Nacional de Treino & EL/GR/LF \\
\hline $13-15$ & CEFAN- RJ & Camp. Brasileiro Sênior* & $\mathrm{EL} / \mathrm{GR} / \mathrm{LF}$ \\
\hline $16-22$ & CT- Rio de Janeiro & Escola Brasileira de técnicos & $\mathrm{EL} / \mathrm{GR} / \mathrm{LF}$ \\
\hline $16-31$ & Sofia- BUL & Treinamento no exterior & $\mathrm{EL} / \mathrm{GR} / \mathrm{LF}$ \\
\hline FEV 2-5 & Sofia- BUL & Torneio "Nikola Petrov" & $\mathrm{EL} / \mathrm{GR} / \mathrm{LF}$ \\
\hline $21-26$ & Colorado Springs-USA & Camp. Panamericano Senior & $\mathrm{EL} / \mathrm{GR} / \mathrm{LF}$ \\
\hline MAR 02-20 & Colorado Springs-USA & Treinamento no exterior & $\mathrm{EL} / \mathrm{GR} / \mathrm{LF}$ \\
\hline $20-25$ & Orlando-USA & Camp. Pan (pré olimpico) & $\mathrm{EL} / \mathrm{GR} / \mathrm{LF}$ \\
\hline ABR2-17 & RJ & Treinamento no Brasil & $\mathrm{EL} / \mathrm{GR} / \mathrm{LF}$ \\
\hline $20-22$ & CEFAN - RJ & Camp. Brasileiro Cadete & $\mathrm{EL} / \mathrm{GR} / \mathrm{LF}$ \\
\hline $23-29$ & Taiyuan-CHN & Torneio Pré olímpico & $\mathrm{EL} / \mathrm{GR} / \mathrm{LF}$ \\
\hline MAI 30/4-06 & Helsinki-FIN & Torneio Pré olímpico & $\mathrm{EL} / \mathrm{GR} / \mathrm{LF}$ \\
\hline $28-7 / 6$ & Havana - CUB & treinamento no exterior (JR) & $\mathrm{EL} / \mathrm{GR} / \mathrm{LF}$ \\
\hline JUN 4-10 & CT- Rio de Janeiro & Escola Brasileira de técnicos & $\mathrm{EL} / \mathrm{GR} / \mathrm{LF}$ \\
\hline $4-5 / 7$ & CEFAN-RJ & Treinamento no Brasil & $\mathrm{EL} / \mathrm{GR} / \mathrm{LF}$ \\
\hline 08-10 & Peten-GUA & Camp. Panamericano Júnior & $\mathrm{EL} / \mathrm{GR} / \mathrm{LF}$ \\
\hline $29-1 / 7$ & Tapiratiba-SP & Camp. Brasileiro Júnior & $\mathrm{EL} / \mathrm{GR} / \mathrm{LF}$ \\
\hline JUL6-8 & Madri - ESP & Grand Prix de Espanha & $\mathrm{EL} / \mathrm{GR} / \mathrm{LF}$ \\
\hline $8-28$ & Madri - ESP & treinamento no exterior & $\mathrm{EL} / \mathrm{GR} / \mathrm{LF}$ \\
\hline $28-5 / 8$ & Marquise- FRA & treinamento no exterior & $\mathrm{EL} / \mathrm{GR} / \mathrm{LF}$ \\
\hline AGO 05-12 & Londres - ENG & Jogos Olímpicos & $\mathrm{EL} / \mathrm{GR} / \mathrm{LF}$ \\
\hline $20-2 / 9$ & CT-RJ & Treinamento no Brasil (JR) & $\mathrm{EL} / \mathrm{GR} / \mathrm{LF}$ \\
\hline SET 3-9 & Pattaya-THA & Campeonato Mundial Junior & $\mathrm{EL} / \mathrm{GR} / \mathrm{LF}$ \\
\hline $05-10$ & Poços de Caldas-MG & Olimpíadas Escolares * & $\mathrm{LF} / \mathrm{EL}$ \\
\hline $25-30$ & Strathcona-CAN & Camp Mundial Feminino & $\mathrm{LF}$ \\
\hline 29 & São Vicente - SP & I Torneio da Juventude & $\mathrm{LF} / \mathrm{EL} / \mathrm{GR}$ \\
\hline OUT2-7 & Helsink-FIN & Camp Mundial Universitário & $\mathrm{EL} / \mathrm{GR} / \mathrm{LF}$ \\
\hline $30-03 / 11$ & Lima- PER & Campeonato Sulamericano & $\mathrm{EL} / \mathrm{GR} / \mathrm{LF}$ \\
\hline NOV2-4 & Porto Alegre-RS & Camp. Bras. Grappling \& Master & $\mathrm{EL} / \mathrm{GR} / \mathrm{LF} / \mathrm{GP}$ \\
\hline 10 & Vitória-ES & II Torneio da Juventude & $\mathrm{LF} / \mathrm{EL} / \mathrm{GR}$ \\
\hline $29-2 / 12$ & Rio de Janeiro - RJ & Copa Brasil Internacional* & $\mathrm{LF} / \mathrm{EL} / \mathrm{GR}$ \\
\hline DEZ1 & Rio de Janeiro - RJ & III Torneio da Juventude & $\mathrm{LF} / \mathrm{EL} / \mathrm{GR}$ \\
\hline $2-9$ & CEFAN- RJ & Campo Internacional de Treino & $\mathrm{EL} / \mathrm{GR} / \mathrm{LF}$ \\
\hline $3-9$ & CEFAN- RJ & Escola Brasileira de técnicos & $\mathrm{EL} / \mathrm{GR} / \mathrm{LF}$ \\
\hline Sem data & Buenos Aires-ARG & Aberto da Argentina & LF/EL/GR \\
\hline
\end{tabular}

Nota: EL - estilo livre; GR - estilo greco-romano e LF - estilo livre feminino.

Acerca da demanda fisiológica em combates competitivos, a análise da estrutura temporal é componente principal para inferências sobre a intensidade, duração e relação esforço/pausa das 
ações. Isso se deve, especialmente, pelo fato das solicitações dos sistemas energéticos durante esportes de combates serem, em grande parte do tempo, supramáximos (Franchini \& Del Vecchio, 2008). Por isso, os conhecimentos sobre quantidades e tipos de ações em combate podem auxiliar nas inferências sobre a contribuição dos sistemas energéticos e na específicidade dos estímulos propostos pelo programa de treinamento (Curby, 2009; Franchini \& Del Vecchio, 2008; LópezGonzález, 2011; López-González \& Alonso, 2011; López-González \& Miarka, 2013; López-Gullón \& Martínez-Abellán, 2011).

\section{Estrutura temporal em combates competitivos de luta olímpica}

Desde os Jogos Olímpicos Modernos de 1896, a modificação das regras da estrutura temporal dos confrontos na luta olímpica fez o tempo limite variar, como demonstra a Tabela 2.

Tabela 2. Variação de tempo de combate e pausa desde a introdução da luta olímpica nos Jogos Olímpicos Modernos (López-Gullón \& Martínez-Abellán, 2011).

\begin{tabular}{ccc}
\hline Data & Períodos de combate & Períodos de pausa \\
\hline 1896 & Sem limite de tempo & Sem intervalo definido \\
1913 & $2 \times 30$ minutos & Sem intervalo definido \\
1913 & $2 \times 20$ minutos & $1 \times 1$ minuto \\
1921 & $1 \times 20$ minutos & Sem intervalo \\
1953 & $2 \times 3$ minutos & $1 \times 1$ minuto \\
1980 & $1 \times 5$ minutos & Sem intervalo \\
1990 & $2 \times 3$ minutos & $1 \times 30$ segundos \\
2000 & $3 \times 2$ minutos & $2 \times 30$ segundos \\
\hline
\end{tabular}

Nilsson, Csergö, Gullstrand, Tveit e Refsnes (2002) indicam que a intensidade e o período de duração dos esforços, pausas e intervalos trazem efeito no desempenho e em respostas fisiológicas à luta olímpica. Logo, como mostra a Tabela 2, o aumento do número de rounds e a redução do tempo de combate e pausa orientaram-na ao aumento de intensidade nas ações. Em suma, algumas investigações, sobre estudos temporais dos combates competitivos, exemplificam como as regras tornam a tentativa de finalizar o combate, pelo touch, antes do tempo oficial uma das principais metas (Tabela 3).

A Tabela 3 também demonstra que cerca de $60 \%$ dos combates competitivos finalizaram no tempo regulamentar determinado pelas regras e que menos de $30 \%$ dos combates acabaram antes desse período. Esses dados são complementares à análise das ações do combate dentro dessa estutura temporal, chamada de Time-Motion Analysis (TMA). Esse é um dos meios usados para investigar demandas fisiológicas e comportamentos técnico-táticos, e pode ser aplicado em melhora do desempenho da luta olímpica (López-González \& Miarka, 2013).

Para esportes de combate, o TMA revela as variações de intermitência intrínsecas em ações técnicas e táticas, considerando as constantes interrupções durante o combate (López-González, 2011; López-González \& Alonso, 2011; López-González \& Miarka, 2013). Na luta greco-romana, Nilsson et al. (2002) mostraram que, no Mundial Sênior Campeonato de 1998, a duração média de trabalho e descanso foi de $37,2 \pm 9,8$ s e $13,8 \pm 6,0$ s, respectivamente. A média de tempo total dos combates foi $427 \mathrm{~s}$ com um tempo total de esforço de $317 \mathrm{~s}$ e pausa de $110 \mathrm{~s}$.

López-González e Miarka (2013) especificaram como foi utilizado o tempo de esforço com a contabilização da frequência de ações na Competição Mundial de 2011 e 2012, em ambos os estilos, através da validação de um modelo de TMA. Esse modelo caracterizou as ações da luta olímpica na seqüência lógica da ocorrência das mesmas durante o combate, como pode ser observado na Tabela 4. A tabela indica que $32 \%$ da frequência de ações ocorreu em disputa de domínio sobre o oponente através da preensão manual, seguido de $29 \%$ em deslocamento sem contato e $14 \%$ em posição parterre - tais resultados revelaram que grande parte do período de combate é usadoem tentativaspara dominar o adversário tanto em pé, como no solo. Dentre as ações que pontuam, $6 \%$ dos ataques foram realizados em pé, 5,6\% em par-terre e apenas 0,8\% em retirada da área. Apesar de pouca frequência, essas ações possuem elevada intensidade e são determinantes para pontuar, o que indica a importância em aprofundar conhecimento sobre essas variáveis técnico-táticas. 
Tabela 3. Estrutura temporal dos combates de luta olímpica em diferentes competições.

\begin{tabular}{|c|c|c|c|}
\hline \multirow{2}{*}{ Autor(es) } & Amostra & Momento & $\%$ \\
\hline & Estilo greco-romano & & \\
\hline \multirow{3}{*}{$\begin{array}{l}\text { Kruszewski et } \\
\text { al. (2011) }\end{array}$} & \multirow{3}{*}{$\begin{array}{l}\text { Combates dos Jogos Olímpicos } \\
\text { de Atlanta } 1996 N=311\end{array}$} & Combates finalizados antes do tempo oficial & $22 \%$ \\
\hline & & Combates finalizados no tempo oficial & $52 \%$ \\
\hline & & Combates finalizados após o tempo oficial & $25 \%$ \\
\hline \multirow{3}{*}{$\begin{array}{l}\text { Kruszewski et } \\
\text { al. (2011) }\end{array}$} & \multirow{3}{*}{$\begin{array}{l}\text { Combates dos Jogos Olímpicos } \\
\text { de Beijing } \\
2008 N=311\end{array}$} & Combates finalizados no 1 round & $2 \%$ \\
\hline & & Combates finalizados entre o 2 e o 3 round & $68 \%$ \\
\hline & & Combates finalizados após o 3 round & $30 \%$ \\
\hline \multirow{3}{*}{$\begin{array}{l}\text { Atan \& } \\
\text { İmamoğlu } \\
(2005)\end{array}$} & \multirow{3}{*}{$\begin{array}{l}21 \text { Combates da Seleção } \\
\text { Nacional da Turquia no } \\
\text { Campeonato Mundial de Patras } \\
2001\end{array}$} & Combates finalizados antes do tempo oficial & $25 \%$ \\
\hline & & Combates finalizados no tempo oficial & $71 \%$ \\
\hline & & Combates finalizados após o tempo oficial & $4 \%$ \\
\hline \multirow{3}{*}{$\begin{array}{l}\text { Atan \& } \\
\text { İmamoğlu } \\
(2005)\end{array}$} & \multirow{3}{*}{$\begin{array}{l}13 \text { Combates da Seleção } \\
\text { Nacional da Turquia } \\
\text { vencedoras no Campeonato } \\
\text { Munidal de Patras } 2001\end{array}$} & Combates finalizados antes do tempo oficial & $12,5 \%$ \\
\hline & & Combates finalizados no tempo oficial & $62,5 \%$ \\
\hline & & Combates finalizados após o tempo oficial & $25,0 \%$ \\
\hline \multirow{4}{*}{$\begin{array}{l}\text { Atan \& } \\
\text { İmamoğlu } \\
(2005)\end{array}$} & \multirow{3}{*}{$\begin{array}{l}334 \text { Combates do Campeonato } \\
\text { Mundial de Patras } 2001\end{array}$} & Combates finalizados antes do tempo oficial & $28 \%$ \\
\hline & & Combates finalizados no tempo oficial & $59 \%$ \\
\hline & & Combates finalizados após o tempo oficial & $11 \%$ \\
\hline & \multicolumn{3}{|l|}{ Estilo livre } \\
\hline \multirow{3}{*}{$\begin{array}{l}\text { Atan \& } \\
\text { İmamoğlu } \\
(2005)\end{array}$} & \multirow{3}{*}{$\begin{array}{l}24 \text { Combates da Seleção } \\
\text { Nacional da Turquia no } \\
\text { Campeonato Mundial de Sofia } \\
2001\end{array}$} & Combates finalizados antes do tempo oficia & $24 \%$ \\
\hline & & Combates finalizados no tempo oficial & $67 \%$ \\
\hline & & Combates finalizados após o tempo oficial & $10 \%$ \\
\hline \multirow{3}{*}{$\begin{array}{l}\text { Atan \& } \\
\text { İmamoğlu } \\
(2005)\end{array}$} & \multirow{3}{*}{$\begin{array}{l}13 \text { Combates da Seleção } \\
\text { Nacional da Turquia } \\
\text { vencedoras no Campeonato } \\
\text { Mundial de Sofia } 2001\end{array}$} & Combates finalizados antes do tempo oficial & $12,5 \%$ \\
\hline & & Combates finalizados no tempo oficial & $50,0 \%$ \\
\hline & & Combates finalizados após o tempo oficial & $37,5 \%$ \\
\hline \multirow{3}{*}{$\begin{array}{c}\text { Atan \& } \\
\text { İmamoğlu } \\
(2005)\end{array}$} & 331 Combates do Campeonato & Combates finalizados antes do tempo oficial & $29 \%$ \\
\hline & \multirow{2}{*}{$\begin{array}{l}\text { Mundial de Sofia } 2001 \\
\text { realizadas por } 47 \text { países }\end{array}$} & Combates finalizados no tempo oficial & $59 \%$ \\
\hline & & Combates finalizados após o tempo oficial & $8 \%$ \\
\hline
\end{tabular}

Tabela 4. Modelo validado para TMA na luta olímpica em frequência e percentual de ocorrência média em lutas olímpicas (López-González \& Miarka, 2013).

\begin{tabular}{lccc}
\hline \multicolumn{1}{c}{ Variável } & Frequência & Percentual & CCI \\
\hline Deslocamento sem contato & 149 & $29,0 \%$ & 0,87 \\
Contato com as palmas & 164 & $31,9 \%$ & 0,84 \\
Controle do oponente por preensão manual & 45 & $8,8 \%$ & 0,22 \\
Penetração & 19 & $3,7 \%$ & 0,83 \\
Técnica em posição de pé & 31 & $6,0 \%$ & 0,44 \\
Saída de área & 4 & $0,8 \%$ & $-0,87$ \\
Preparação em par-terre & 39 & $7,6 \%$ & 0,91 \\
Técnica em par-terre & 29 & $5,6 \%$ & 0,60 \\
Controle da posição de touch & 9 & $1,8 \%$ & 0,99 \\
Interrupção & 19 & $3,7 \%$ & 0,99 \\
Pausa entre rounds & 6 & $1,2 \%$ & 0,98 \\
\hline Nota: Sumári da descricão
\end{tabular}

Nota: Sumário da descrição das ações do modelo: Deslocamento sem contato - momento em que os atletas não possuem nenhum contato; Contato com as palmas - momento em que ocorre o contato entre os lutadores, mas sem tração; Tipo de controle do oponente por preensão manual- momento em que ocorre tração do oponente, através do contato realizado pela preensão das mãos do atleta em partes do corpo adversário (mais comuns em membros superiores, região torácica e dorsal); Penetração - momento em que ocorre investida com intuido de alcançar a região dos membros inferiores; Técnica de posição em pé - momento que que ocorre técnica de projeção ou domínio das costas em pé; Saída de área - momento em que ocorre a saída da área; Preparação em par-terre - momento em que ocorre a situação de par-terre. Técnica em par-terre momento em que ocorre a aplicação de técnica durante o par-terre; Controle da posição de touch- momento em que ocorre a retenção no solo; Interrupção - momento em que o confronto é interrompido; Pausa entre rounds- intervalo entre os rounds (González-López \& Miarka, 2013) 


\section{Estrutura técnica e tática dos combates competitivos de luta olímpica}

A análise técnico-tática é um meio utilizado para compreender o modo pelo qual as habilidades esportivas são desempenhadas, o que pode fornecer informações para promover o desempenho na modalidade (López-González \& Miarka, 2013; Miarka et al., 2010). Por exemplo, em combates realizados nos Jogos Olímpicos de Londres 2012, cerca de 30\% das vitórias obtidas não necessitaram de entrada técnica (Figura 2) (FILA, 2015).

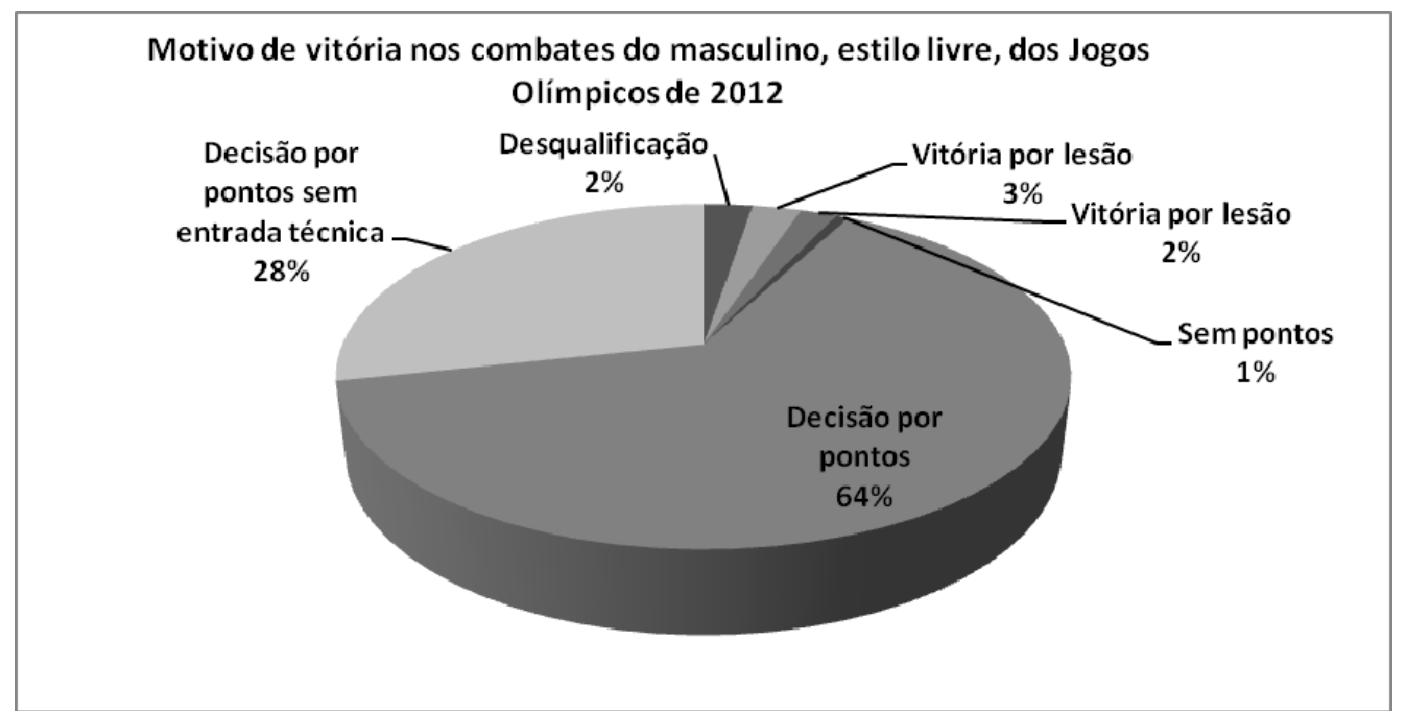

Figura 2. Gráfico segundo o tipo de vitória nos combates do masculino, estilo livre, dos Jogos Olímpicos de Londres 2012 (dados adaptados de Tünnemann, 2013).

A Figura 2 revela que as conquistas dos Jogos Olímpicos de Londres 2012 foram obtidas através de domínio parcial do adversário ou/e ponto por punição adversária ou/e por saída de área. Esses dados, assim como as demais investigações sobre análises da estrutura técnica e tática da luta olímpica possuíam como foco principal verificar a eficácia das ações pela quantidade de pontos gerados por minuto de combate (López-González, 2010, 2011; Tünnemann, 2011a,b,c). Tünnermann $(2011 \mathrm{a}, \mathrm{b}, \mathrm{c})$ demonstrou esses resultados ao longo de 34 anos e como isso pôde ser influenciado pelo contexto das regras nos três estilos (Figura 3).

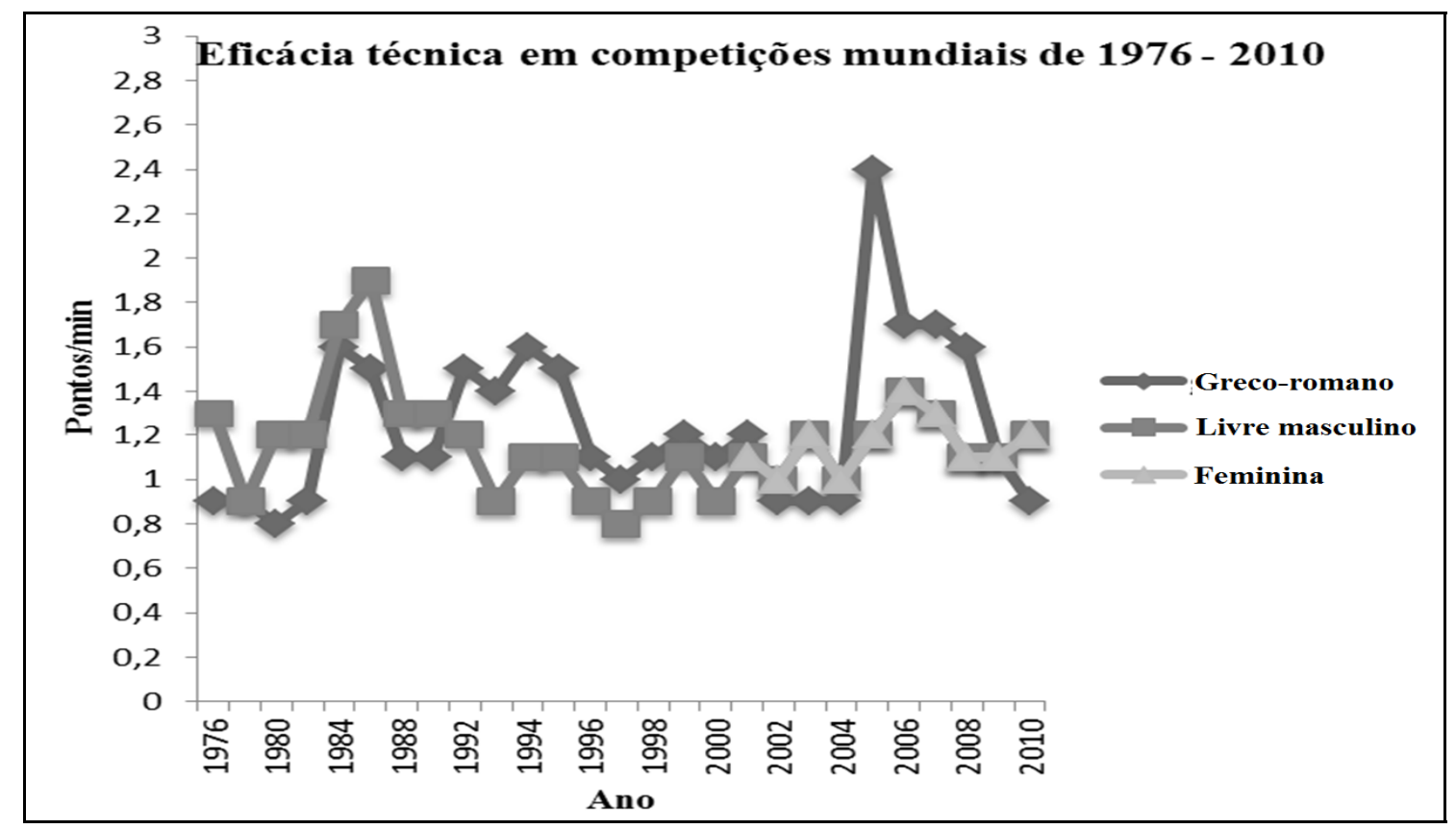

Figura 3. Gráfico da quantidade de pontos conquistados por minuto por atletas ganhadores de combates mundiais nos três estilos de luta olímpica de 1976 até 2010 (dados de Tünnermann, 2011a,b,c). 
A Figura 3 mostra dois picos acentuados de pontos por minuto, em 1984 no estilo grecoromano e livre, e em 2005 nos três estilos. Especialmente no estilo greco-romano, houve aumento na eficácia durante o Campeonato Mundial de Budapeste 2005, no qual os atletas ganhadores atingiram 2,4 pts/min. Em contrapartida, nesse mesmo estilo, as demais mudanças ocasionaram queda da eficácia para apenas 0,9 pts/min durante o Campeonado Mundial de Moscou 2010, o qual ocorreu logo após as mudanças nas regras de 2009. Tünnermann (2011b) sugere que tais modificações nas regras aumentaram a atitude defensiva dos atletas durante o combate e a busca por pontuação no momento de clinch. Durante os combates de greco-romano, grande parte dos estudos apontam os Takedowns como as técnicas mais eficazes em competições mundiais de 2009 até 2011. Para o mesmo período, na luta olímpica feminina e estilo livre, as técnicas de pé/perna são as mais eficazes, seguida dos ataques de Takedown, como pode ser observado na Tabela 5.

Tabela 5. Frequência de pontuação por minuto de combate em combates de Competições Mundiais entre 2009 e 2011 (estilo greco-romano, estilo livre y feminina).

\begin{tabular}{|c|c|c|c|c|c|c|}
\hline \multirow{2}{*}{ Autor(es) } & \multicolumn{2}{|c|}{ Estilo greco-romano } & \multicolumn{2}{|l|}{ Estilo livre } & \multicolumn{2}{|l|}{ Feminina } \\
\hline & Ação & Efic. & Ação & Efic. & Ação & Efic. \\
\hline & Takedowns & 0,17 & Ataques de perna/pé & 0,27 & Ataques de perna/pé & 0,60 \\
\hline Tünnemann & Projeções & 0,27 & Takedowns & 0,13 & Takedowns & 0,20 \\
\hline$(2011 a, b, c)$ & Rolê & 0,23 & Projeções & 0,02 & Projeções & 0,08 \\
\hline Censo do & Turnover & 0,00 & Rolê & 0,08 & Rolê & 0,12 \\
\hline Campeonato & Lifts & 0,13 & Turn over & 0,08 & Turnover & 0,30 \\
\hline Mundial em & Contra-ataque & 0,07 & Contra-ataque & 0,13 & Contra-ataque & 0,08 \\
\hline \multirow[t]{2}{*}{2009} & Fora de área & 0,04 & Fora da área & 0,08 & Fora da área & 0,04 \\
\hline & & & Clinch & 0,15 & Clinch & 0,03 \\
\hline & Takedowns & 0,24 & Ataques de perna/pé & 0,34 & Ataques de perna/pé & 056 \\
\hline Tünnemann & Projeções & 0,25 & Takedowns & 0,21 & Takedowns & 0,56 \\
\hline$(2011 a, b, c)$ & Rolê & 0,31 & Projeções & 0,02 & Projeções & 0,21 \\
\hline Censo do & Turnover & 0,11 & Rolê & 0,18 & Rolê & 0,09 \\
\hline Campeonato & Lifts & 0,00 & Turnover & 0,30 & Turnover & 0,11 \\
\hline Mundial em & Contra-ataque & 0,10 & Contra-ataque & 0,08 & Contra-ataque & 0,50 \\
\hline \multirow[t]{2}{*}{2010} & Fora de área & 0,10 & Fora da área & 0,06 & Fora da área & 0,14 \\
\hline & Penalidade & 0,06 & Clinch & 0,07 & Clinch & 0,03 \\
\hline & Takedowns & 0,12 & Ataques de perna/pé & 0,49 & Ataques de perna/pé & 0,46 \\
\hline Tünnemann & Projeções & 0,21 & Takedowns & 0,13 & Takedowns & 0,27 \\
\hline$(2011 a, b, c)$ & Rolê & 0,10 & Projeções & 0,02 & Projeções & 0,15 \\
\hline Censo do & Turnover & 0,05 & Rolê & 0,08 & Rolê & 0,10 \\
\hline Campeonato & Lifts & 0,00 & Turnover & 0,08 & Turnover & 0,29 \\
\hline Mundial em & Contra-ataque & 0,03 & Contra-ataque & 0,13 & Contra-ataque & 0,20 \\
\hline \multirow[t]{2}{*}{2011} & Fora de área & 0,14 & Fora da área & 0,08 & Fora da área & 0,07 \\
\hline & & & Clinch & 0,15 & Clinch & 0,10 \\
\hline
\end{tabular}

Nota: Efic. $=$ Eficácia em pontos por minuto

Além do índice de pontos por minuto das principais formas de pontuar, indicadas na Tabela 5 , diferentes investigações demonstraram a frequência absoluta das ações que pontuaram em combates competitivos do estilo livre e greco-romano (Tabela 6).

A Tabela 6 demonstra que cerca de $20 \%$ das técnicas utilizadas no estilo livre são de pé/perda e que, tanto no estilo livre, quanto em alguns estudos do estilo greco-romano, o Rolê está entre as principais ações ofensivas realizadas no solo. Além desses dados, é importante observar que os ataques são resultado de um conjunto de ações prévias realizadas durante o combate. Diante disso, recentes investigações observaram o conjunto de ações prévias ao ataque (López-González, 2010, 2011). López-González (2010) verificou padrões táticos de lutadores participantes do Campeonato Mundial 2010, em estilo livre. Para isso, o autor dividiu a sequência tática em dois momentos: i) inicial/preparatório, e ii) técnica. As combinações táticas mais utilizadas foram:

- deslocamento longo sem contato $\rightarrow$ ii) Takedown" ( $r=0,667)$.

- deslocamento curto com contato $\rightarrow$ ii) Ataque de perna/pé $(r=0,503)$.

- deslocamento curto sem contato $\rightarrow$ ii) Takedown $(r=0,554)$. 
Tabela 6. Ações que pontuaram em competições nos estilos greco-romano e livre.

\begin{tabular}{|c|c|c|c|}
\hline \multirow[t]{2}{*}{ Autor(es) } & Amostra & Ação & $\begin{array}{l}\text { Frequência durante as } \\
\text { competições }\end{array}$ \\
\hline & \multicolumn{3}{|l|}{ Estilo greco-romano } \\
\hline Curby (2005) & $\begin{array}{l}\text { Censo do Campeonato } \\
\text { Estadual Universitário de } \\
\text { Illinois } 2005\end{array}$ & $\begin{array}{l}\text { Takedowns } \\
\text { Escapes } \\
\text { Touch } \\
\text { Reversão } \\
\text { Touch parcial } \\
\text { Penalizações }\end{array}$ & $\begin{array}{l}148(46 \% \text { do total }) \\
71(22 \% \text { do total }) \\
42(13 \% \text { do total }) \\
32(10 \% \text { do total }) \\
19(6 \% \text { do total }) \\
10(3 \% \text { do total })\end{array}$ \\
\hline $\begin{array}{l}\text { Curby, } \\
\text { Dzierdzic \& } \\
\text { Fraser (2008) }\end{array}$ & $\begin{array}{l}\text { Principais técnicas de } \\
\text { projeção que pontuaram } \\
\text { em164 combates Jogos } \\
\text { Olímpicos de Beijng } 2008\end{array}$ & $\begin{array}{l}\text { Pushouts } \\
\text { Arm Drag } \\
\text { Snap Down } \\
\text { Contra-ataques } \\
\text { High Dive } \\
\text { Técnica de braço (3 pontos) }\end{array}$ & $\begin{array}{l}31(34 \% \text { do total }) \\
10(11 \% \text { do total }) \\
10(11 \% \text { do total }) \\
7(8 \% \text { do total }) \\
6(7 \% \text { do total }) \\
6(7 \% \text { do total })\end{array}$ \\
\hline $\begin{array}{l}\text { Atan \& } \\
\text { İmamoğlu } \\
(2005)\end{array}$ & $\begin{array}{l}\text { Campeonato Mundial } \\
2001\end{array}$ & $\begin{array}{l}\text { Rolê } \\
\text { Tying Salto } \\
\text { Contra-ataque do Rolê } \\
\text { Throw Back } \\
\text { Queda de ombro }\end{array}$ & $\begin{array}{l}16(30 \% \text { do total }) \\
8(15 \% \text { do total }) \\
5(9 \% \text { do total }) \\
4(7 \% \text { do total }) \\
4(7 \% \text { do total })\end{array}$ \\
\hline $\begin{array}{l}\text { Atan \& } \\
\text { İmamoğlu } \\
\text { (2005) }\end{array}$ & $\begin{array}{l}\text { Seleção Turca no } \\
\text { Campeonato Mundial de } \\
2001\end{array}$ & $\begin{array}{l}\text { Rolê } \\
\text { Suplê } \\
\text { Contra-ataque de Rolê } \\
\text { Queda de ombro }\end{array}$ & $\begin{array}{l}17(33 \%) \\
5(10 \%) \\
5(10 \%) \\
4(8 \%)\end{array}$ \\
\hline & Estilo livre & & \\
\hline $\begin{array}{l}\text { Atan \& } \\
\text { İmamoğlu } \\
\text { (2005) }\end{array}$ & $\begin{array}{l}\text { Campeonato Mundial } \\
2001\end{array}$ & $\begin{array}{l}\text { Ataques de perna/pé } \\
\text { Rolê } \\
\text { Double leg } \\
\text { Throw Back }\end{array}$ & $\begin{array}{l}22(36 \% \text { do total }) \\
15(17 \% \text { do total }) \\
4(6 \% \text { do total }) \\
1(1,5 \% \text { do total })\end{array}$ \\
\hline $\begin{array}{l}\text { Atan \& } \\
\text { İmamoğlu } \\
\text { (2005) }\end{array}$ & $\begin{array}{l}\text { Seleção Turca no } \\
\text { Campeonato Mundial de } \\
2001\end{array}$ & $\begin{array}{l}\text { Ataques de perna/pé } \\
\text { Rolê } \\
\text { Head Drag } \\
\text { Double leg } \\
\text { Contra-ataque de Gravata }\end{array}$ & $\begin{array}{l}17(24 \% \text { do total }) \\
14(20 \% \text { do total }) \\
9(13 \% \text { do total }) \\
7(10 \% \text { do total }) \\
6(8,5 \% \text { do total })\end{array}$ \\
\hline
\end{tabular}

Com relação à luta feminina, López-González (2010) computou 1.923 ataques durante o Campeonato Mundial de 2009. Desse total, 593 obtiveram pontuações e 794ações ofensivas marcaram pontos técnicos. A Tabela 7 mostra as 15 situações com maior quantidade de pontos obtidos.

Tabela 7. As 15 situações táticas com maior quantidade de pontos convertidos no Campeonato Mundial de Luta Olímpica Feminina 2009, em Herning (López-González, 2010a).

\begin{tabular}{lccc}
\hline \multicolumn{1}{c}{ Situação } & Frequência & $\begin{array}{c}\text { Frequência de } \\
\text { pontuação }\end{array}$ & $\begin{array}{c}\text { Pontos } \\
\text { totais }\end{array}$ \\
\hline Takedown com apoio externo do joelho & 286 & 52 & 59 \\
Takedown em Clinch & 58 & 42 & 45 \\
Retirada da zona de combate & 50 & 35 & 39 \\
Bloqueio & 34 & 34 & 47 \\
Finta por tipo de domínio e Takedown & 193 & 31 & 47 \\
Takedown direto, sem ajoelhar & 81 & 30 & 36 \\
Esquiva por tipo de domínio e Takedown & 88 & 27 & 42 \\
Contra-ataque & 31 & 25 & 40 \\
Penetração entre as pernas e Fireman & 68 & 21 & 50 \\
Combinação de Takedowns & 37 & 37 & 22 \\
Takedown no calcanhar & 56 & 15 & 22 \\
Torsões (hip flip) & 34 & 14 & 28 \\
Extensão de tronco sobre a cabeça do oponente e takedown & 47 & 14 & 47 \\
Takedown em ambas as pernas com mãos abertas & 58 & 11 & 17 \\
\hline
\end{tabular}


As informações da Tabela 7, sobre a freqüência das diferentes situações competitivas que pontuam, trazem importantes implicações táticas. Além disso, podem auxiliar no entendimento das variáveis neuromotoras envolvidas em cada ação utilizada no combate. Os estudos publicados durante os últimos anos, especialmente no último ciclo olímpico 2009 - 2012 têm ajudado a conceituar e aperfeiçoar um conjunto de variantes de modo a tornar as análises técnico-táticos de confrontos úteis para determinar um conjunto de tendências cujo conhecimento poderia obter grande relevância para o treinamento desenvolvido no mundo, e em especial para reforçar esse esporte em torno da oportunidade de crescimento que tem implícita (a recomendação do COI para cair luta livre dos Jogos Olímpicos de 2020, lançada desde o ano de 2013) (López-González, 2014).

Em maio de 2013, a FILA anunciou mudanças drásticas nas regras dos três estilos Olímpicos (Tünnemann, 2013). 0 estilo greco-romano experimentou as mudanças mais significativas em uma tentativa de neutralizar a baixa freqüência de pontuação e da redução nas técnicas de variedades que prevaleceram durante o ciclo olímpico de 2009-2012. Este fenômeno foi documentado por Tünnemann através de uma comparação longitudinal da qualidade técnica dos combates nos Torneios Olímpicos de Sidney 2000 e Londres 2012 (Tünnemann, 2013). 0 índice de eficácia em Londres foi de 0,8 pontos por minuto, ou seja, a mais baixa produtividade técnica nesse período (Tünnemann, 2012). Felizmente, Tünnemann (2013) recente relata conseqüências favoráveis na seqüência da implementação destas mudanças de regras nos quatro principais campeonatos do ano de 2013. Os pontos por minuto aumentaram significativamente no Mundo, por exemplo, nos Campeonatos de Cadet (1,6 pts / min), júnior (3,5 pts / min) e categorias de Seniores (1,4 pts / min), assim como o 27th Universíada de Verão (1,5 pts / min) (Tünnemann, 2013; López-González, 2014). 0 consenso geral é que as estratégias defensivas pouco atraentes que prevaleceram durante pelo menos os últimos dois ciclos olímpicos desapareceram e deram lugar a um mais ofensivo e ativo confronto, especialmente na greco-romana, com uma maior quantidade de pontos técnicos (López-González, 2014; Tünnemann, 2013).

Os dados obtidos a partir da análise exploratória iniciais de López-González (2014) confirmam o impacto positivo das alterações de regras na luta greco-romana, na qual as ações de ataque na fase do combate em pé ou em posição de Par-terre aumentaram significativamente em comparação com Jogos Olímpicos de 2012, quando apenas 33,3\% dos lutadores participantes (45 de 135 concorrentes) tinham executado um ou mais ações de ataque em ambas as posições (LópezGonzález, 2014; Tünnemann, 2013).

A análise dos Lutadores revelou maiores valores de variação das nas ações $(0,27 \pm 0,08)$ e de ações ofensivas determinantes em Par-Terre $(0,97 \pm 0,06)$, por exemplo, técnicas o Gutwrenches e movimentos derivados e elevadores como o Suplex. Pode-se inferir que a maior variação está relacionada com a freqüência de ações ao longo do combate (López-González, 2014; Tünnemann, 2013).

López-González (2014) indica que os lutadores que atingiram o top 5 colocações em 2013 os Campeonatos Mundiais de Seniores em Luta greco-romana são caracterizados com uma atividade técnica e tática alta em pé e em posição de par-terre. López-González (2014) baseado em seis variáveis discriminantes, os lutadores foram agrupados em 8 grupos, com destaque para quatro estratégias de grupos que incluíram medalhistas de ouro:

- Grupo I: Altamente ofensivo em ambas as posições, ganhando ativamente a posição de vantagem em par-terre com base em ataques de risco moderado (takedown com passagem por trás e penetrações) e, em seguida, marcando com muitos gutwrench diversificados e com suplex e variantes dessa elevação.

- Grupo II: Em pé, com atividade contra-ofensiva, de curta distância, marcando pontos moderadamente na posição par-terre.

- Grupo III: Com uma elevada variedade e freqüência de ataques em pé (incluindo várias ações com alto risco), com freqüência moderada de ataques em par-terre.

- Grupo IV: Baixa eficácia na posição de pé, técnicas de baixo risco (takedowns e passagem por trás, bloqueios contra-ofensivos, pushouts, quase sem movimentos de penetração) e freqüência moderada em par-terre, com o menor número de atletas agrupados nesse conjunto. 


\section{Limitações e futuras pesquisas}

Apesar das importantes contribuições para compreensão sobre as demandas dos combates da luta olímpica, poucos trabalhos foram realizados até hoje sobre ações técnico-táticas e demandas fisiológicas nos combates de luta olímpica. Por sua vez, as pesquisas mostradas nessa revisão possuem limitações. A principal delas, inerente ao método de pesquisa utilizado, é a impossibilidade de generalização dos resultados que são sobre atletas de elite (López-González, 2014; Tünnemann, 2013). Por isso, a partir das evidências encontradas podem-se derivar hipóteses e sugestões para futuras pesquisas com lutadores iniciantes e intermediários para verificar as características técnico-táticas nesses níveis competitivos que almejam obter informações para serem utilizadas em planejamento de treinamentos e/ou preparação física específica diante da demanda competitiva.

Quanto ao método, análises notacionais possuem limitações que incluem: i) o tempo para gravar com precisão manualmente os confrontos, e; ii) a necessidade da verificação da validade interna das variáveis do protocolo a partir da análise de concordância entre e intra-analistas (López-González \& Miarka, 2013) para garantir confiabilidade ao ponto de refletir informações táticas precisas e inferências fisiológicas relacionadas com a luta olímpica (Barbas, et al., 2011; López-González, 2014; Tünnemann, 2013). Ainda não foram feitos experimentos sobre como a aplicação prática das informações relacionadas com as demandas do combate competitivo contido nos estudos pode refletir em treinamento contextualizado e nos conseqüentes resultados dos próximos combates competitivos, o que sugere pesquisas sobre o feedback gerado com as informações da análise notacional para aquisição, retenção e transferência de habilidades técnicotáticas contextualizadas de acordo com a demanda dos combates da luta olímpica.

\section{Conclusão}

O objetivo dessa revisão foi apresentar a caracterização dos combates de luta olímpica nos estilos livre, feminino e greco-romana. Essas informações possuem importante aplicação prática, uma vez que revelam conhecimento para práticas contextualizadas em treinamentos técnico-táticos e preparação física e/ou avaliações com ações análogas aos combates da luta olímpica. 0 conhecimento técnico-tático traz implicações teóricas para futuros estudos que considerem situações táticas críticas e as demandas geradas durante os confrontos. Por exemplo, durante os combates de greco-romano, grande parte dos estudos aponta os Takedowns como as técnicas mais eficazes em competições mundiais de 2009 até 2011. Para o mesmo período, na luta olímpica feminina e estilo livre, as técnicas de pé/perna são as mais eficazes, seguida dos ataques de Takedown. Sendo que as últimas análises mostram que as ações ofensivas determinantes são aplicadas em pé e em par-terre, especialmente técnicas de Gutwrenches e movimentos derivados do Suplex. Esses conhecimentos, além de possibilitarem maior especificidade no treinamento, orientam a aplicação prática em intervenções preventivas às possíveis lesões conseqüentes das ações técnico-táticas.

\section{References}

Atan, T., \& İmamoğlu, O. (2005). Competition analysis of World Greco-Roman and World Free-style wrestling championships. International Journal of Performance Analysis in Sport, 5(1), 3140.

Barbas, I., Fatouros, I. G., Douroudos, I. I., Chatzinikolaou, A., Michailidis, Y. , Draganidis, D., Jamurtas, A. Z., Nikolaidis, M. G., Parotsidis, C., Theodorou, A. A., Katrabasas, I., Margonis, K., Papassotiriou, I., \& Taxildaris, K. (2011). Physiological and performance adaptations of elite greco-roman wrestlers during a one-day tournament. European Journal of Applied Physiology, 111(7), 1421-1436.

Barna, T. (2013). Elite wrestlers orientation to tactical information. International Journal of Wrestling Science, 3(1), 10-16.

Cipriano, N. (1993). A technical-tactical analysis of freestyle wrestling. Journal of Strength \& Conditioning Research, 7(3), 133-140. 
Confederação Brasileira de Lutas Associadas, CBLA (2013). Confederação Brasileira de Lutas Associadas. Recuperado em 2 de janeiro 2013, de http://cbla.com.br

Curby, D. (2009). Scoring Patterns in High School Wrestling 2009. Recuperado em 2 de janeiro 2013 de http://www.curbywrestling.com/sport\%20science.htm

Curby, D. (2005). USOEC Wrestling Testing Program 2005. Recuperado em 12 de janeiro 2013 de http://www.curbywrestling.com/grecometrika.htm

Curby, D., Dzierdzic, S., \& Fraser, S. (2008). Analysis of the 2008 Olympic Greco-roman Wrestling Competition. Recuperado em 4 de janeiro 2013 de http://www.curbywrestling.com/pdf/Beijing\%20Games\%20GR\%20Scoring\%20Analysis.p df

FILA - Federation Internationale Des Luttes Associess (2015). Federation Internationale Des Luttes Associess. Recuperado em 10 de janeiro 2015 de http://www.fila-wrestling.com

Franchini, E., \& Del Vecchio, F. B. (2008). Preparação física para atletas de judô. São Paulo: Phorte.

Gomes A. C. (2002). Treinamento Desportivo Estruturação e periodização. Porto Alegre: Artmed.

Horswill, C. A. (1992). Applied physiology of amateur wrestling. Sports Medicine, 14(2), 114-143.

Kirov, P., \& Makavéev, O. (2010). Preparation Sportive Du Lutteur. Sofia: Fédération bulgare de lutte NCA Presse.

Kraemer, W. J., Fry, A. C., Rubin, M. R., Triplett-McBride, T., Gordon, S. E., Koziris, L. P., Lynch, J. M., Volek, J. S., Meuffels, D. E., Newton, R. U., \& Fleck, S. J.(2001). Physiological and performance responses to tournament wrestling. Medicine Science in Sports and Exercise, 33(8), 13671378.

Kruszewski, A., Zarczuk, P., Kruszewski, M., Kuzmicki, S., Jagiello, W., \& Blach, W. (2011). Directions of changes of technical and tactical skills by wrestlers free style within 12 years, 1996-2008. Journal of Combat Sports and Martial Arts, 2(2), 117-123.

Lafon, M. (2008). FILA Master Degrees. Lausanne: Fédération Internationale des Luttes Associées.

López-González, D. (2010). Caracterización de las combinaciones técnico-tácticas en posición de pie realizadas con efectividad en el Campeonato del Mundo Senior de Lucha Femenil 2009, Revista de Ciencias del Ejercicio, 6, 18-21.

López-Gonzalez, D. (2011). Tactical Means Classification in Freestyle and Female Wrestling. XIX FDD Educacion Fisica, Deportes y Ciencias Aplicadas, Sto. Domingo, DO.

López-González, D. (2014). Technical-tactical performance in greco-roman wrestling: analysis of 2013 Senior World Championships through mutivariate analysis. International Journal of Wrestling Science, 4(1), 95-110.

López-González, D. E., \& Miarka, B. (2013). Reliability of a New Time-motion Model Based on Technical-tactica Interactions for Wrestling Combats. International Journal of Wrestling Science, 3, 21-34.

López-González, D., \& Alonso, A. (2011). Determinantes del rendimiento técnico-táctico en lucha estilo libre y femenil del campeonato del mundo. Revista de Ciencias del Ejercicio, 7, 156158.

López-Gullón, J. M., \& Martínez-Abellán, A. (2011). Evolución del reglamento oficial de luchas olímpicas y sus consecuencias sobre las capacidades condicionales y habilidades técnicotáticas. E-Balonmano.com. Revista de Ciencias del Deporte, 7 (suppl.), 39-44.

Miarka, B., \& Conceição, R. A. (2012). Pedagogia do ensino da luta olímpica em escolas. Em E. Franchini \& F. B. Del Vecchio, (Eds.), Ensino de lutas: Reflexões e Propostas de Programas (pp183-208). São Paulo: Scortecci.

Miarka, B., Julio, U. F., Del Vecchio, F. B., Calmet, M., \& Franchini, E. (2010). Técnica y Táctica en Judo: una revisión. Revista de Artes Marciales Asiáticas, 5(1), 91-112.

Nilsson, J., Csergö, S., Gullstrand, L., Tveit, P., \& Refsnes, P. E. (2002). Work-time profile, blood lactate concentration and rating of perceived exertion in the 1998 Greco-Roman wrestling World Championship. Journal of Sports Sciences, 20(11), 939-945.

Platonov, V. N. (2004). Teoria geral do treinamento desportivo olímpico. Porto Alegre: Artmed.

Rhea, M. R., Ball, S. D., Phillips, W. T., \& Burkett, L. N. (2002). A comparison of linear and daily undulating periodized programs with equated volume and intensity for strength. Journal of Strength Conditioning Research, 16(2), 250-255.

Tünnermann, H. (2011a). Annual Scoring Analysis of World Championships by Greco-Roman. International Journal of Wrestling Science, 1, 41-52. 
Tünnermann, H. (2011b). Annual Scoring Analysis of World Championships by Freestyle. International Journal of Wrestling Science, 1, 21-31.

Tünnermann, H. (2011c). Annual Scoring Analysis of World Championships by Women's Freestyle. International Journal of Wrestling Science, 1, 32-40.

Tünnemann, H. (2012). Analysis of the Olympic Games London and the Olympic Cycle 2008 - 2012 in Greco Roman Wrestling. Recuperado em 4 janeiro 2013 de http://www.filaofficial.com/images/FILA/documents/stages/2012/Analysis_of_the_OG_Lo ndon_and_cycle_2008_2012_GR. pdf.

Tünnemann, H. (2013). Evolution and Adjustments for the New Rules in Wrestling. International Journal of Wrestling Science, 3 (2), 94-104.

Yoon, J. (2002). Physiological Profiles of Elite Senior Wrestlers. Sports Medicine, 32(4), 225-233.

\section{Author's biographical data}

Bianka Miarka (Brasil), Ex-atleta internacional da Seleção de Remo e da Seleção Brasileira de Judô, com títulos internacionais. É pós-doutoranda na Universidade Federal de Pelotas, doutora em Biodinâmica pela Escola de Educação Física e Esporte da Universidade de São Paulo (2014). Possui graduação em Ciência do Esporte (2005) e especialização em Treinamento Esportivo (2007) pela Universidade Estadual de Londrina e mestrado em Educação Física pela Escola de Educação Física e Esporte da Universidade de São Paulo. (2010). Possui como foco de pesquisa trabalhos em lutas, esportes, treinamento físico, análise de movimento, tecnologia e bioestatística. E-mail:miarkasport@hotmail.com. 